\title{
The existences of frame wavelet set
}

\author{
Wan-She Li \\ School of Mathematics and Information Science \\ Shaanxi Normal University \\ Xi'an, China \\ liwsh@snnu.edu.cn
}

\begin{abstract}
In this paper, Let $A$ be a $d \times d$ real expansive matrix, it mainly discusses the existences of frame wavelet set, we discuss the characterization of frame wavelet sets in $R^{d}$, and several examples are presented, in order to deepen the understanding of frame wavelet set, which gives the two related theorems; we try to use an equivalent condition to describe frame scale sets, and give an equivalent description about a normalized frame scale set.
\end{abstract}

Keywords- frame; frame wavelet set; frame scale set;

\section{INTRODUCTION}

Wavelet analysis is a new mathematical analysis method in the nineteen eighties, has repeatedly caused many academic research climax. Now, wavelet analysis has been widely used in various fields, especially in image processing and signal processing with a spurt of achievement. Although many people feel magical on the application of wavelet; people do not know much about construction of the wavelet set, based on this, this article begins with the frame wavelet set. The expansion and shift system of an orthogonal wavelet composes a standard orthogonal basis, the expansion and translation system of a frame wavelet composes of a frame, the frame and the standard orthogonal basis compared with more of mobility, This makes wavelet and frame lessons from each other, promote each other in two areas of mathematics, and the research of framework for wavelet research has opened up a way.

In [1] we characterize the definition of the framework and Fourier transform of function, by [5] we know affine system and a characterization of MRA, in [8]-[9] we studied the characterization of wavelet set in the different space, we can know relevant knowledge about wavelet in [10] - [13]. In [2]-[4] we give characterization of the frame wavelet set, and gives two examples about the judgment of frame wavelet set; We obtain some necessary conditions and sufficient conditions of scaling function in [7], and in this paper we present a equivalent characterization of scaling function.

\section{PREMILINARY}

A collection of element $\left\{x_{j}\right\}_{j \in Z}$ in a Hilbert space $H$ is called a frame if there exist constant $A$ and $B$, $0<A \leq B<\infty$, such that

\author{
Hong-Xia Zhao \\ School of Mathematics and Information Science \\ Shaanxi Normal University \\ Xi'an China \\ 714273197@qq.com
}

$$
A\|f\|^{2} \leq \sum_{j \in Z}\left|<f, x_{j}>\right|^{2} \leq B\|f\|^{2}, \forall f \in H
$$

When $A=B$, we say that the frame is tight.

When $A=B=1$, we say the frame is normalized tight. Any orthonormal basis in a Hilbert space is a normalized tight frame but not versa.

Let $A$ be a $d \times d$ real invertible matrix. It induces a unitary operator $D_{A}$ acting on $L^{2}\left(R^{d}\right)$ defined by

$$
\left(D_{A} f\right)(t)=|\operatorname{det} A|^{\frac{1}{2}} f(A t), \forall f \in L^{2}\left(R^{d}\right), t \in R^{d}
$$

The matrix $A$ is called expansive if all its eigenvalues have modulus greater than one. The operator $D_{A}$ corresponding to a real expansive matrix $A$ is called an A-dilation operator. In an analogous fashion, a vectors in $R^{d}$ induce a unitary $T_{s}$ defined by

$$
\begin{aligned}
& \left(T_{s} f\right)(t)=f(t-s), f \in L^{2}\left(R^{d}\right), t, s \in R^{d} \\
& \text { If } f \in L^{1}\left(R^{d}\right) \cap L^{2}\left(R^{d}\right), \\
& \text { then }(\hat{f})(s)=\frac{1}{(2 \pi)^{\frac{d}{2}}} \int_{R^{d}} e^{-2 \pi i<s, t>} f(t) d t
\end{aligned}
$$

Where $\langle s, t>$ denotes the real inner product. Now let $E$ be a Lebesgue measurable set with finite measure and let $\psi \in L^{2}\left(R^{d}\right)$ be defined by $\hat{\psi}=(2 \pi)^{-d / 2} \chi_{E}$. For any $\hat{f} \in L^{2}\left(R^{d}\right)$, let $H_{E} \hat{f}$ be the following formal summation:

$$
H_{E} \hat{f}=\sum_{n \in Z, l \in Z^{d}}<\hat{f}, \hat{D}_{A}^{n} \hat{T}^{l} \hat{\psi}>\hat{D}_{A}^{n} \hat{T}^{l} \hat{\psi}
$$

Let $E_{d}$ denote the set of all expanding matrices $A$, Now we fix an arbitrary matrix $A \in E_{d}$, for a function $\psi \in L^{2}\left(R^{d}\right)$, we will consider the affine system $\Psi$ defined by

$\Psi=\left\{\psi_{j, k}(x) \mid \psi_{j, k}(x)=2^{\frac{j}{2}} \psi\left(A^{j} x-k\right), j \in Z, k \in Z^{d}\right\}$

Let $G$ be a Lebesgue measurable set of finite measure. 
For any $l \in Z^{d}$, let $I_{l}$ denote the d-cube $[0,1)^{d}+l$.

For any subset $G$ of $R^{d}$, define:

$$
\tau(G)=\bigcup_{l \in Z^{d}}\left(G \cap 2 \pi I_{l}-2 \pi\right) \text {. }
$$

If the above is a disjoint union, we say that $G$ is translation equivalent to $\tau(G)$, which is a subset of $2 \pi I_{0}$, where $I_{0}$ is the unit d-cube $[0,1)^{d}+l$. If $G$ and $F$ are translation equivalent to the same subset in $2 \pi I_{0}$, then we say $G$ and $F$ are translation equivalent. This defines an equivalent relation and is denoted by $\doteq$. Let $\mu(\cdot)$ be the Lebesgue measure. It is clear that $\mu(G) \geq \mu(\tau(G))$. The equivalent holds if and only if $G=\tau(G)$. If $G=F$, then $\mu(G)=\mu(F)$. Two point $x, y \in G$ are said to be translation equivalent if $x-y=2 \pi$ for some

$l \in Z^{d}$.The translation redundancy index of a point $x$ in $G$ is the number of elements in its equivalent class. We write $G(\tau, k)$ for the set of all points in $G$ with translation redundancy index $k$. In general, $G(\tau, k)$ could be an empty set, proper subset of $G$, or the set of $G$ itself. For $k \neq m, G(\tau, k) \cap G(\tau, m)=\theta$,

so $G=G(\tau, \infty) \cup\left(\bigcup_{n \in N} G(\tau, n)\right)$.

Let $E$ be a point set in $R^{d}$. Throughout this article, we will use $\chi_{E}$ to denote the characteristic function of $E$, let $\theta$ be an empty set.

Definition 2.1 Let $\left\{V_{j}\right\}_{j \in Z}$ be a sequence of closed subspaces of $L^{2}\left(R^{d}\right)$ satisfying:
(a) $V_{j} \subset V_{j+1}$;
(b) $\overline{\cup_{j \in Z} V_{j}}=L^{2}\left(R^{d}\right)$;
(c) $\cap_{j \in Z} V_{j}=\{0\}$;
(d) $f \in V_{j} \Leftrightarrow f(2 \cdot) \in V_{j+1}, j \in Z$;
(e)There exists a function $\phi \in V_{0}$ such that

$\left\{T_{k} \phi: k \in Z\right\}$ is an orthonormal basis of $V_{0}$. Then $\left\{V_{j}\right\}_{j \in Z}$ is called an MRA and the function $\phi$ from (e) is called a scaling function.

\section{FRAME WAVELAE SET}

In order to study frame wavelet set, we need the following theorems and lemmas:

Lemma 3.1 Let $M, N$ be Lebesgue measurable sets of finite measurable in $R^{d}$ such that

$$
\tau(M)=\tau(N) \subset[0,2 \pi)^{d} .
$$

If $M=M(\tau, 1), N=N(\tau, 1)$, then for any $2 \pi$ periodical function, we have

$<f(s) \chi_{M},(2 \pi)^{\frac{-d}{2}} e^{-i<l, s>}>=<f(s) \chi_{N},(2 \pi)^{\frac{-d}{2}} e^{-i<l, s>}>$

For the sake of convenience, we denote $<f(s),(2 \pi)^{\frac{-d}{2}}|\operatorname{det}|^{\frac{-k}{2}} e^{-i<l,\left(A^{\prime}\right)^{-k} s>} \cdot \chi_{\left(A^{\prime}\right)^{k} E}>$ by $a_{k l}$, to avoid confusion, we have keep in mind that $a_{k l}$ depends on $E$ and $f$ under discussion.

Lemma 3.2 Let $E$ be a Lebesgue measurable set in $R^{d}$ with finite positive measure. The following statements are equivalent:

(i) There exists a constant $a>0$ such that for any

$k \in \mathrm{Z}, \sum_{l \in \mathrm{Z}^{d}}\left|a_{k l}\right|^{2} \leq a\left\|f \cdot \chi_{\left(A^{\prime}\right)^{k} E}\right\|^{2}$ for all

$f \in L^{2}\left(R^{d}\right)$

(ii) There exists $M>0$ such that $\mu(E(\tau, m))=0$ for all $m \geq M$.

Lemma 3.3 Let $E$ be a Lebesgue measurable set with finite measure. Then the following statements are equivalent:

(i) $H_{E}$ defines a bounded linear operator in $L^{2}\left(R^{d}\right)$, that is, $H_{E} \hat{f}$ converges in $L^{2}\left(R^{d}\right)$ for any $\hat{f} \in L^{2}\left(R^{d}\right)$ and $\left\|H_{E} \hat{f}\right\| \leq b\|\hat{f}\|$ for some constant $b>0$.

(ii) There exists a constant $c>0$ such that

$$
\begin{aligned}
& \sum_{n \in Z, l \in Z^{d}}\left|<\hat{f}, \hat{D}_{A}^{n} \hat{T}^{l}(2 \pi)^{-d / 2} \chi_{E}>\right|^{2} \leq c\|\hat{f}\|^{2}, \\
& \forall \hat{f} \in L^{2}\left(R^{d}\right) .
\end{aligned}
$$

(iii) There exists a constant $M>0$ such that $\mu(E(\delta, m))=0$ and $\mu(E(\tau, m=0))$ for any $m \geq M$.

We outline the main results obtained in this paper below. Theorem 3.4 Let $E$ be a Lebesgue measurable set with finite measure. Then $E$ is a frame wavelet set if

(i) $\bigcup_{n \in Z}\left(A^{\prime}\right)^{n} E(\tau, 1)=R^{d}$;

(ii) There exists $M>0$ such that $\mu(E(\delta, m))=0$ and $\mu(E(\tau, m))=0$ for any $m>M$.

Proof of Theorem 3.4 By the proof of Lemma 3.3, we have $\left|<H_{E} f, f>\right| \leq M^{\frac{5}{2}}\|f\|$.

On the other hand,

$\sum_{j=1}^{m} f_{m j}^{k} \cdot \chi_{\left(A^{\prime}\right) E(\tau, m)}=\sum_{l \in Z^{d}} a_{k l} \hat{D}_{A}^{k} \hat{T}^{l}(\sqrt{2 \pi})^{-d} \chi_{E(\tau, m)}$

(with $a_{k l}$ depending on $E(\tau, m)$ not $E$ ), hence 


$$
<\sum_{j=1}^{m} f_{m j}^{k} \cdot \chi_{\left(A^{\prime}\right)^{k} E(\tau, m)}, f>=\sum_{l \in Z^{d}}\left|a_{k l}\right|^{2} \geq 0
$$

It follows that

$$
\begin{aligned}
& <H_{E} f, f>=\sum_{k \in Z} \sum_{m=1}^{M} \sum_{j=1}^{m}<f_{m j}^{k} \cdot \chi_{\left(A^{\prime}\right)^{k} E(\tau, m)}, f> \\
& \geq \sum_{k \in Z}<f \cdot \chi_{\left(A^{\prime}\right)^{k} E(\tau, 1)}, f> \\
& =\int_{R^{d}}|f|^{2}\left(\sum_{k \in Z} \chi_{\left(A^{\prime}\right)^{k} E(\tau, 1)}\right) d s \geq\|f\|^{2},
\end{aligned}
$$

since $\sum_{k \in Z} \chi_{\left(A^{\prime}\right)^{k} E(\tau, 1)} \geq 1$, by the given condition.

Theorem 3.5 If $E=E(\tau, 1), \bigcup_{n \in Z}\left(A^{\prime}\right)^{n} E(\tau, 1)=R^{d}$ and there exists $1 \leq k_{1} \leq k_{2}$ such that $\mu(E(\delta, m))=0$ for $m<k_{1}$ and $m>k_{2}, \mu\left(E\left(\delta, k_{1}\right)\right) \mu\left(E\left(\delta, k_{2}\right)\right) \neq 0$, then $E$ is an frame wavelet set whose corresponding frame has a lower bound $k_{1}$ and an upper bound $k_{2}$.

Proof of Theorem 3.5. If $E=E(\tau, 1)$ and

$$
\begin{aligned}
& \bigcup_{n \in Z}\left(A^{\prime}\right)^{n} E(\tau, 1)=R^{d} \text {, then } \\
& H_{E} f=\sum_{n \in Z} H_{E}^{n} f=\sum_{n \in Z} f \cdot \chi_{\left(A^{\prime}\right)^{n} E},
\end{aligned}
$$

Assume that $\bigcup_{n \in Z} \chi_{\left(A^{\prime}\right)^{n} E}=k$,

then $<H_{E} f, f>=<\sum_{n \in Z} f \cdot \chi_{\left(A^{\prime}\right)^{n} E}, f>$ $=\int_{R^{d}}|f|^{2} \sum_{n \in Z} \chi_{\left(A^{\prime}\right)^{n} E} d s=\|f\|^{2}$

for all $f \in L^{2}\left(R^{d}\right)$, since the fact $\mu(E(\delta, m))=0$, $m<k_{1}, m>k_{2}$, that is $k_{1}\|f\|^{2} \leq k\|f\|^{2} \leq k_{2}\|f\|^{2}$, we can gain the desired result $k_{1} \leq k \leq k_{2}$. Then $E$ is an frame wavelet set whose corresponding frame has a lower bound $k_{1}$ and an upper bound $k_{2}$.

Theorem 3.6 If $E=E(\tau, 1)$ and $\mu(E(\tau, k)) \neq 0$ for some $k>1$, then $E$ is not a frame wavelet set.

Proof of Theorem 3.6 By lemma $3^{[3]}$, if $E=E(\tau, 1)$, then $H_{E} f=f$. Assume that $E$ is a frame wavelet set, $g=\chi_{E^{(1)}(\tau, k)}, h=\chi_{E^{(2)}(\tau, k)}, f_{1}=g+h, f_{2}=g-h$.

Since $k>1$,

$\mu(E(\tau, k)) \neq 0, \mu\left(E^{(1)}(\tau, k)\right)=\mu\left(E^{(2)}(\tau, k)\right)$, then $\left\|f_{1}\right\|^{2}=\left\|f_{2}\right\|^{2}$, that is $<H_{E} f_{1}, f_{1}>=<H_{E} f_{2}, f_{2}>$.
Because $<H_{E} f_{1}, f_{1}>=<H_{E} g+H_{E} h, g+h>$ $=<g, g>+<H_{E} g, h>+<H_{E} h, g>+<h, h>$, $<H_{E} f_{2}, f_{2}>=<H_{E} g-H_{E} h, g-h>$ $=<g, g>-<H_{E} g, h>-<H_{E} h, g>+<h, h>$, $<H_{E} g, h>$ and $<H_{E} h, g>$ are both positive, we can gain that $<H_{E} f_{1}, f_{1}>\neq<H_{E} f_{2}, f_{2}>$, this contradicts $\left\|f_{1}\right\|^{2}=\left\|f_{2}\right\|^{2}$, then $E$ is not an frame wavelet set. In the following, we give several examples.

Example 3.7 Let $E=\left[-\frac{3 \pi}{2},-\frac{\pi}{2}\right) \cup\left(\frac{\pi}{4}, \pi\right]$,

Then $\bigcup_{n \in Z} 2^{n} E(\tau, 1)=R, \mu(E(\delta, m))=0$,

$\mu(E(\tau, m))=0, \forall m>1$, by the Theorem 3.4,

so $E$ is an frame wavelet set.

Example 3.8 Let

$E=[\pi, 2 \pi) \times[\pi, 2 \pi) \cup[\pi, 2 \pi) \times[3 \pi, 4 \pi)$, then $E=E(\tau, 2)$, by the Theorem 3.6, $E$ is an frame wavelet set.

\section{FRAME SCALE SET}

Theorem 4.1 Let $G$ be a bounded closed set in $R^{d}$. Then there exists a frame scaling function $\phi$ with $\operatorname{supp} \hat{\phi}=G$ if and only if

(a) $G \subset 2 G$;

(b) $\bigcup_{j \in Z} 2^{m} G=R^{d}$;

(c) $\tau(G) \subset T^{d}$;

(d) $\left(G \backslash \frac{1}{2} G\right) \cap\left(\frac{1}{2} G+2 \pi k\right)=\theta,\left(k \in R^{d}\right)$.

Theorem 4.2 Let $G$ be a bounded closed set and $\left\{T_{k} \phi: k \in \mathrm{Z}\right\}$ be the normalized tight frame of $V_{0}$ with supp $\hat{\phi}=G$. If the following statements

(i) $\forall B \subset G$, $\left|\int_{B} \hat{\phi}(\omega) d \omega\right|^{2}=\mu(B)$;

(ii) $\operatorname{supp} \hat{\phi}(\cdot-l) \cap \operatorname{supp} \hat{\phi}(\cdot-k)=\theta$, $\left(l, k \in \mathrm{Z}^{d} . l \neq k\right)$;

(iii) $\bigcup_{n \in Z^{d}} \operatorname{supp} \hat{\phi}(\cdot-n)=R^{d}$ hold; We have

(a) $G \subset 2 G$;

(b) $\bigcup_{m \in Z} 2^{m} G=R^{d}$;

(c) $G=G(\tau, 1)$;

(d) $\left(G \backslash \frac{1}{2} G\right) \cap\left(\frac{1}{2} G+2 \pi k\right)=\theta,\left(k \in Z^{d}\right)$.

Lemma 4.3 Let $\phi \in L^{2}\left(R^{d}\right)$ and a closed set $G$ satisfy the following: 
(a) $\operatorname{supp} \hat{\phi}=G$;

(b) $\hat{\phi}(2 \omega)=H(\omega) \hat{\phi}(\omega)$ a.e. $\omega \in R^{d}\left(H \in L^{\infty}\left(T^{d}\right)\right)$;

(c) $\sum_{n \in Z^{d}}|\hat{\phi}(\omega+2 \pi n)|^{2}=1$ a.e. $\omega \in T^{d} \backslash N$;

(d) $\bigcup_{m \in \mathrm{Z}} 2^{m} G=R^{d}$;

then $\phi$ is a scaling function.

Lemma 4.4 Let $G$ be a bounded closed set and $\left\{T_{k} \phi: k \in \mathrm{Z}\right\}$ be the normalized tight frame of $V_{0}$ with supp $\hat{\phi}=G$. The following statements are equivalent:

(i) $\operatorname{supp} \hat{\phi}(\cdot-l) \cap \operatorname{supp} \hat{\phi}(\cdot-k)=\theta$, $\left(l, k \in \mathrm{Z}^{d}, l \neq k\right)$.

(ii) $<\phi(\cdot-l), \phi(\cdot-k)>=\delta_{l, k},\left(l, k \in \mathrm{Z}^{d}\right)$.

Proof of Lemma 4.3,

(i) $\Rightarrow$ (ii) $\operatorname{supp} \hat{\phi}(\cdot-l) \cap \operatorname{supp} \hat{\phi}(\cdot-k)=\theta$

$\left(l, k \in \mathrm{Z}^{d}, l \neq k\right)$,

So $<\hat{\phi}(\cdot-l), \hat{\phi}(\cdot-k)>=(2 \pi)^{-d / 2} \delta_{l, k}$.

By Paiseval equation, $\langle\phi(\cdot-l), \phi(\cdot-k)\rangle=\delta_{l, k}$.

(ii) $\Rightarrow$ (i)

Since $<\phi(\cdot-l), \phi(\cdot-k)>=\delta_{l, k},\left(l, k \in \mathrm{Z}^{d}\right)$;

by Paiseval equation ,

$<\phi(\cdot-l), \phi(\cdot-k)>$

$=\left(\frac{1}{2 \pi}\right)^{-d / 2}<\hat{\phi}(\cdot-l), \hat{\phi}(\cdot-k)>=\left(\frac{1}{2 \pi}\right)^{-d / 2} \delta_{l, k}$,

So $k \neq l,\left(k, l \in Z^{d}\right)$,

$\operatorname{supp} \hat{\phi}(\cdot-l) \cap \operatorname{supp} \hat{\phi}(\cdot-k)=\theta$.

Corollary 4.5 Let $G$ be a bounded closed set and $\left\{T_{k} \phi: k \in \mathrm{Z}^{d}\right\}$ be the normalized tight frame of $V_{0}$ with $\operatorname{supp} \hat{\phi}=G$, If

(i) $\forall B \subset G,\left|\int_{B} \hat{\phi}(\omega) d \omega\right|^{2}=\mu(B)$;

(ii) $<\phi(\cdot-l), \phi(\cdot-k)>=\delta_{l, k}, \quad\left(l, k \in \mathrm{Z}^{d}\right)$;

(iii) $\bigcup_{n \in Z^{d}} \operatorname{supp} \hat{\phi}(\cdot-n)=R^{d}$ hold,

then (a) $G \subset 2 G$;

(b) $\bigcup_{m \in Z} 2^{m} G=R^{d}$;

(c) $G=G(\tau, 1)$;

(d) $\left(G \backslash \frac{1}{2} G\right) \cap\left(\frac{1}{2} G+2 \pi k\right)=\theta,\left(k \in Z^{d}\right)$.

\section{REFERENCES}

[1] C.K. Chui, An Introduction to Wavelets, Academic Press, Boston, 1992. [2] X. DAI, Y. DIAO, Q. DU, Frame wavelet sets in $R$, Proceedings of the American Mathematical Society, 2001 129(7): 2045-2055.

[3] X. Dai, Y. Diao, Q. Gu, D.Han. Frame wavelet sets in $R^{d}$ [J]. Journal of Computational and Mathematics,155(2003):69-82.

[4] Dai X, Larson D, Spleegle D. Wavelet sets in $R^{d}$ [J]. J. Fourier Anal Appl. 1997,3(4):451-456.

[5] Zhanwei Liu, Guoen Hu, Guoen Hu, Guogchang Wu. Frame scaling function sets and frame wavelet sets in $R^{d}$ [J].Chaos, Solitions and Fractals, 40(2009):2483-2490.

[6] Zhuihua Zhang*, Naoki Saito, Ring-like structures of frequency domain of wavelet, Appl.Comput.harmon.Anal.29(2010 )18-29.

[7] Zhang ZS. Supports of Fourier transform of scaling functions. Appl Comput Harmon Anal 2007,22(2):141-156.

[8] Dai X, Larson D, Speegle D. Wavelet sets in $R^{d}$.J Fourier Anal Appl. 1997;3(4): 451-456.

[9] X. Dai, S. Lu, Wavelets in subspaces, Mich .J. Math. 43(1996) 81-89.

[10] Speegle D. The s-elementary wavelet sets in are pash-connected. Proc Am Math Soc. 1999;127(1); 223-233.

[11] El Naschie MS. Hilbert Frock and Cantorian spaces in the quantum two-slit experiment. Chao, Solition \& Fractals. 2006; 27(1): 39-42.

[12] El Naschie MS. A review of E-infinity theory and the mass spectrum of high energy particle ghysics. Chao, Solition \& Fractals. 2004; 19(1):209-236.

[13] El Naschie MS. A guide to machematics of E-infinity Cantorian spacetime theory. Chao, Solition \& Fractals. 2005; 25(5):955-964. 